\title{
Fourniers gangrene-A case report and literature review
}

\author{
A.Agarwal ${ }^{1}$, A.Karmacharaya ${ }^{2}$, S.Hirachan², W.K.Belokar ${ }^{3}$ \\ ${ }^{1}$ Mch Resident ${ }^{3}$ Prof and HOD Department of Urology, ${ }^{2}$ Resident Department of Surgery, College of Medical Sciences, \\ Teaching Hospital, Bharatpur, Chitwan, Nepal
}

\begin{abstract}
Fournier's gangrene(FG) is a rare, necrotising fasciitis of the genitals and perineum with occasional cranial extension to the abdominal wall caused by a mixture of aerobic and anaerobic microorganisms. The complications of this synergistic infection are multiple organ failure and death. Due to the aggressive nature of this condition, early diagnosis is crucial. Treatment involves extensive soft tissue debridement and broad-spectrum antibiotics. Despite appropriate therapy, mortality is high. We report a case of a gentleman who presented with gangrenous and necrotic penile, scrotal and perineal areas. Patient was successfully managed by extensive soft tissue debridement, broad spectrum antibiotics and skin grafting.
\end{abstract}

Key words : Fournier's gangrene, necrotizing fascitis, tissue, debridement, skin grafting.

\section{Introduction}

FG is an uncommon, rapidly progressive infection of the male genital, perineal and perianal regions with occasional cranial extension to the abdominal wall. It is characterized by a synergistic, necrotising fasciitis leading to the thrombotic occlusion of small subcutaneous vessels and the development of gangrene. ${ }^{1}$ The first case was described by Baurienne in 1764 but the condition was named by Fournier in 1883 who reported the cases of five men with the condition with no apparent etiology. Infection most commonly arises from the skin, urethra, or rectal regions. There are two types. Type I is due to a mixture of aerobic and anerobic organisms usually following an abdominal operation or associated with diabetes

Correspondence: A. Agarwal

E-mail: draaman@gmail.com mellitus. ${ }^{2}$ Type II is due to Group A Streptococcus synergistic with a second organism (Staphylococcus aureus, coliforms, Bacteroides spp.). ${ }^{2,3}$ The majority of patients with FG are immunocompromised ${ }^{3,4}$ Malignant disease, obesity, diabetes mellitus, peripheral vascular disease, local trauma, urethral stricture and perianal disease have been cited as the main predisposing factors. ${ }^{5}$ Due to the aggressive nature of this condition, early diagnosis is crucial. Treatment involves extensive soft tissue debridement and broadspectrum antibiotics. Despite appropriate therapy, mortality is high. ${ }^{6}$ We report case of fournier's gangrene (FG) and review it's causes and management.

\section{Case report}

A 66 year-old-gentleman presented with fever and pain with blackish discolouration of penis and scrotal skin for 2 days followed by blister formation and ulceration. 
A.Agarwal et al. Fourniers gangrene-A case report and literature review

History revealed that he was suffering from LUTS for last 2 years with recurrent UTI due to grade IV BPH. Perineal examination revealed the entire penile and scrotal skin to be gangrenous and necrotic ulcer. In addition, there was purulent stinking secretion. Patient was non diabetic. There was no history of trauma or instrumentation or any sepsis in the genito-perineal area. No other co-morbid diseases were known and there was no previous history of any drug allergies and local trauma or operation.

On general examination, patient had fever and vital signs showed tachycardia with normal Blood pressure. He was conscious, blood pressure was 110/70 mmHg and pulse rate was 122/min. Complete blood picture revealed leucocytosis and neutrophilia with raised ESR and normal $\mathrm{Hb} \%$. Complete Urine examination, random blood sugar, blood urea and serum creatinine was within normal limits.. USG of Abdomen and Pelvis showed grade IV BPH (66gm) with thickening of urinary bladder wall. Local examination revealed foul smelling blackish discolouration of entire Peno-scrotal area extending to suprapubic region without any clear line of demarcation.

The patient was dehydrated. He was admitted at College of medical sciences and broad spectrum antibiotics, ceftriaxone, amoxycillin, metronidazole and intravenous fluid resuscitation were started parenterally. Patient was diagnosed as case of FG. Emergency extensive debridement of soft tissue with copius wound irrigation was done. After three days once the infection was controlled exposed testis were transposed to medical side of thigh bilaterally. Culture of discharge from the infected skin grew E.coli, Streptococcus pyogenes, organisms sensitive to Ceftriaxone, Aztreonam, Cefoperazone, Amikacin. After debridement(fig-1) and regular dressing of penoscrotal raw area, when the bed was having good granulations, unexpanded meshed split thickness skin grafting done over penis(fig-2), taken from anterior thigh. Both the testies were transposed to medial side of thigh and secondary closure of suprabubic wound was done(fig-2). Patient made uneventful recovery post operatively. As the cause, we thought, was related to urinary tract, transvesical open prostatectomy was done in the same sitting.

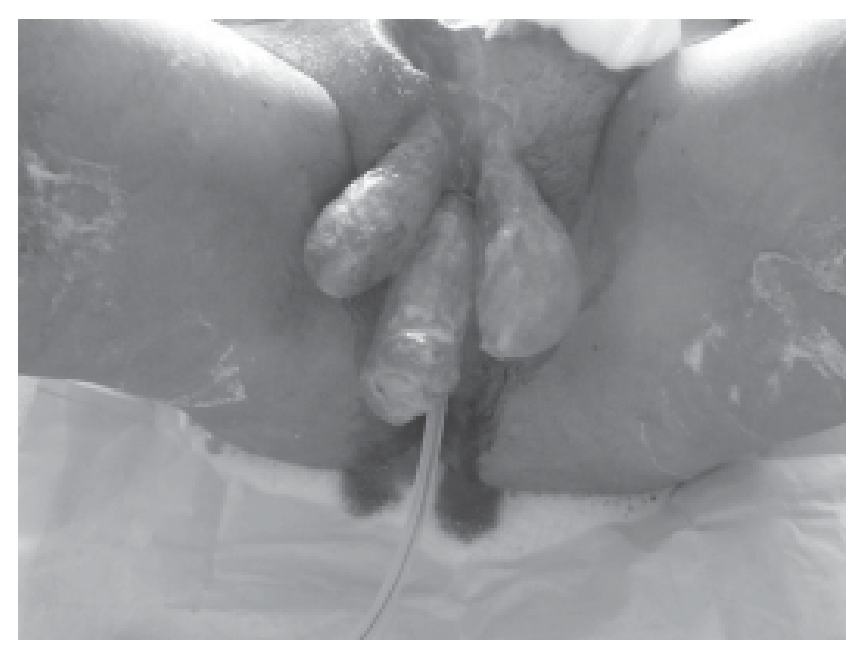

Fig. 1: Extensive debridement done for penile and scrotal area

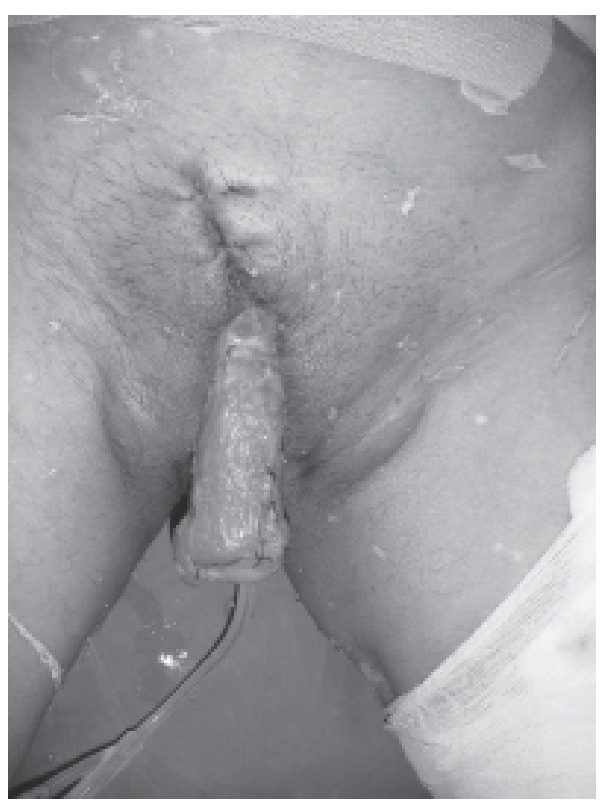

Fig. 2: Medial transposition of both testes with split thickness penile skin graft. 
Journal of College of Medical Sciences-Nepal,2011,Vol-7,No-3

\section{Discussion}

Necrotising fasciitis is a fulminant, rapidly progressive spreading infection. Widespread tissue destruction and necrosis of fat and fascia with overlying secondary necrosis of skin are characteristic of the infection. Jean Alfred Fournier, a French venerologist, described a subgroup of necrotizing fasciitis in $1883 .{ }^{8}$ Originally, it was thought to be an idiopathic infection of healthy males. However, modern use of the term Fournier's gangrene has been expanded to include all perineal necrotizing infections in both men and women. ${ }^{7}$ The majority of infections documented occur in diabetic men aged 50 to 70 years. ${ }^{8}$ It is a serious condition characterised by cellulitis of the scrotal skin leading to subcutaneous necrosis, all of which are preceded by subcutaneous crepitations. The testes are unaffected. ${ }^{3}$ In a large population based epidemiologic study, 1641 males and 39 females with FG identified. Cases represented less than $0.02 \%$ of hospital admissions. The overall incidence was 1.6/100,000 males, which peaked in males who were 50 to 79 years old. The overall case fatality rate was $7.5 \%{ }^{9}$

The illness begins with a prodromal period of genital discomfort and pruritis followed by sudden

onset of perineal pain ${ }^{5,7}$ However, as the gangrene progresses this pain is replaced by anaesthesia. ${ }^{7}$ Initially, the skin may appear to be normal and the extent of the subdermal gangrene may not be apparent. ${ }^{3,7}$ Progression through the fascial layers results in deep tissue necrosis and gangrenous skin changes, resulting in drainage of the affected area and demarcation between viable and dead tissue., $\mathrm{A}$ by-product of anaerobic metabolism is the formation of crepitus, composed of hydrogen, hydrogen sulphide, nitrogen and nitrous oxide, which may be detected on x-ray, thus indicating the presence of dead tissue. ${ }^{7,8}$ All of these signs are associated with foul odour. There is rapid development of severe toxaemia with associated signs, for example, pyrexia with or without hypothermia, leukocytosis, thrombocytopenia, raised blood urea and nitrogen, tachycardia, hypotension and reduced urine output. ${ }^{7,8}$ This may occur in just a few hours progressing to organ failure and death.

One of the most important causes of this infection is poor hygiene. Usually it is due to a less aggressive, more routine infectious process through some point of entry involving the colon, urinary tract, prostate or anorectal area. ${ }^{5,7}$ It is secondary to polymicrobial infection by aerobic and anaerobic bacteria with a synergistic action. Candida albicans has also been reported as the primary microorganism. ${ }^{10}$ The most commonly isolated microorganism in both male and female patients was $\mathrm{E}$ coli. ${ }^{11}$ The aetiology is identified in $95 \%$ of cases. The source of infection is either cutaneous, urogenital or colorectal areas. On the basis of Cakmak study on the 65 cases the most common etiology was hemorrhoidectomy in male and perianal abscess in female patients. ${ }^{11}$ In a series of 45 patients with FG, Basoglu et al reported that the most common etiology was perirectal abscess followed by scrotal carbuncle and thrombosed hemorrhoid. ${ }^{12}$. Korkout et al reported that the etiology was identifiable in 95\% of cases, diabetes mellitus being a predisposing factor in $55.6 \% .{ }^{13}$ In our case Prostatic infection seems to be the only responsible cause. It can also proceed from cellulitis or traumatic injury involving cutaneous structures in the perianal region. ${ }^{7}$ Obstetric events such as vaginal deliveries with episiotomies and Caesarean section, carcinoma of the large intestine, hematological malignancies, severe neutropenia and burns have also been implicated. ${ }^{7}$ Predisposing factors for the 
A.Agarwal et al. Fourniers gangrene-A case report and literature review

development of necrotising soft tissue infections include: poor perfusion, hypertension, renal insufficiency, trauma, diabetes mellitus, malnutrition, immune suppression, cigarette smoke, intravenous drug abuse, obesity and spinal cord injury ${ }^{7,14}$ Old age itself is not a predisposing factor; however, elderly patients with poor self care and poor nutritional status are more susceptible to FG and have a poorer prognosis. ${ }^{5}$

Diagnosis is usually clinical and the role of diagnostic imaging is limited. A full blood count is appropriate which in this case will reveal anaemia and leukocytosis. Renal profile is impaired in septic shock. Coagulopathies, such as disseminated intra-vascular coagulopathy, secondary to sepsis/septic shock may also be seen. A mid-stream urine sample is taken to exclude urinary tract infection. The source of infection should be investigated by whatever means necessary. An intravenous pyelogram, barium enema, sigmoidoscopy and/or cystoscopy may be appropriate and the underlying cause should be treated. ${ }^{3}$ Tissue biopsies and pus are sent for culture and sensitivity.

These infections are a surgical emergency; a delay in treatment is associated with a significantly higher mortality rate. Radical debridement should be carried out, aggressively removing all necrotic tissue and any marginally perfused tissue. Debridement should be continued until all remaining tissues are adherent and viable. ${ }^{7}$ As a rule, the penis, testes, bladder and rectum are spared if possible. Spirnak et al. reported that patients who undergo multiple operations have greater mortality. ${ }^{15}$ Appropriate intravenous broadspectrum antibiotics which are effective against both anaerobic gram-negative and facultative aerobic organisms are important therapeutic adjuvants, but are not substitutes for proper surgical debridement. Empirical therapy is given initially which can then be modified on the basis of culture and sensitivity tests. The patient's results should be reviewed daily and creatinine phosphokinase (CPK) levels should be obtained to monitor for myonecrosis. Nutritional parameters such as albumin, prealbumin and transferrin should be monitored to assure proper caloric intake. The use of hyperbaric oxygen therapy is debatable as its therapeutic value is unclear. ${ }^{7}$ The wound is closed later with skin grafting. FG is a life threatening illness requiring emergency surgery, despite which the patient may still not survive. Mortality rates as high as 20 to 30 percent have been described in some studies. ${ }^{4}$ Certain factors influencing the survival of these patients, primarily relating to the patient's metabolic status and the extent of the disease, were evaluated by Laor et al. from which the FG severity index (FGSI) was formulated. ${ }^{16}$ In the FGSI, nine parameters are measured and the degree of deviation from normal is graded from 0 to 4 . The individual values are summed to obtain the FGSI score (FGSIS). Using a FG severity index threshold of 9 (sensitivity $71.4 \%$, specifity $90 \%$ ) there was a 96\% survival rate in patients with a FG severity index of less than 9 and a 46\% mortality rate in those with a Fournier gangrene severity index of 9 or greater $(\mathrm{p}=$ 0.001, OR 22,95\% CI 3.5-139.7). ${ }^{17}$

However, early diagnosis and treatment and the arrest of the gangrene at an early stage markedly improve outcome. The most common error in the management of these lesions is delayed or inadequate surgical debridement. Other factors associated with increased mortality in necrotizing infections are advanced age, co-existent systemic sepsis and development of organ failure. Co-morbid conditions such as cancer, renal 
insufficiency and congestive heart failure also contribute to an increased mortality rate. ${ }^{8}$

\section{Conclusion}

Since the infection is distributed secretly and rapidly through fascial plains, clinical findings in early period may be considered the "tip of the iceberg". Although FG is a rare disease, it has a high mortality rate. Early diagnosis, immediate radical surgical debridement (s), use of appropriate antibiotics, and long-term hemodynamic support when necessary are required to reduce FG's high mortality rate. It seems that there are no major differences between male and female patients in the characteristics of the condition. ${ }^{11}$ Good management is based on aggressive debridement, broad spectrum antibiotics and intensive supportive care. ${ }^{18}$ Hyperbaric oxygen therapy and reconstructive procedures may be needed in the course of treatment process. ${ }^{19}$ The FG severity index remains an objective and simple method to quantify the extent of metabolic aberration at presentation in patients with FG.

\section{Acknowledgement}

I acknowledge Dr Sudeep Raj K.C. for secretarial assistance.

\section{References}

1. S.P. Patankar, S.K.Lalwani. Fournier's gangrene. JIndian Paediatr 2004;41:511.

2. P. Kumar ,M. Clarke, eds. Clinical Medicine. 5th ed.Edinburgh: WB Saunders, 2002:66-7.
3. G.R. McLatchie, D.J. Leaper, eds. Oxford Handbook of Clinical Surgery. 2nd ed. Oxford UK: Oxford University Press, 2003;53:890.

4. W. Pawlowski, M.Wronski, I.W.Krasnodebski. Fournier's gangrene. Pol Merkuriusz Lek 2004; 17(97):85-7.

5. C.O.Yeniyol, T.Suelozogen, M.Arslan,et al Fournier’s gangrene: Experience with 25 patients and the use of Fournier's gangrene severity index score. Urology 2004;64(2):218-22.

6. R. Muqim. Necrotising fasciitis: management and outcome. J Coll Physicians Surg Pak 2003;13(12):711-4.

7. J.L.Cameron, ed. Current Surgical Therapy. 8th ed. Philadelphia: Elsevier Mosby, 2004;1079-85.

8. R.N. Upport, H.M.Levy, P.H.Patel. Case 54: Fournier’s Gangrene. Radiology 2003;226:115-7.

9. M.D.Sorensen, J.N.Krieger, F.P.Rivara, et al. Fourniers gangrene: population based epidemiology and outcomes. J Urol 2009; 181(5):2120-6.

10. M.Temiz, M.Cetin, A.Aslan. Fourniers gangrene caused by Candida albicans. Mikrobiyol Bul 2008 ; 42(4):707-711.

11. A.Cakmac, V.Genc, C.Akyol, et al. Fourniers gangrene: is it scrotal gangrene. Advanced Therapeutics 2008 ; 25(10):1065-74.

12. M.Basoglu, I.Ozbey, S.S.Atamanalp, et al. Management of Fourniers gangrene: review of 45 cases. Surg today 2007, 37(7):558-563.

13. M.Korkut, G.Icoz, M.Dayangac, et al. Outcome analysis in patients with Fourniers gangrene: report of 45 cases. Dis Clon Rectum 2003 , 46(5):649-652.

14. S.Vaidyanathan, B.M.Soni, P.L.Hughes, et al. Necrosis of the scrotum (Fournier's gangrene) in a spinal cord injury patient - a case study. BMC Fam Pract 2002;3; 20 
A.Agarwal et al. Fourniers gangrene-A case report and literature review

15. J.P.Spirnak, M.I.Resnick, N.Hampel. Fournier's gangrene: report of 20 patients. J Urol 1984;131:289-92.

16. E.Laor, L.S.Palmer, B.M.Toila, et al. Outcome prediction in patients with Fournier's gangrene. J Urol $1995 ; \mathbf{1 5 4}: 89-92$

17. A.T.Corcoran, M.C.Smaldone, E.P.Gibbons,et al. Validation of the Fourniers gangrene severity index in a large contemporary series. J Urol 2008 , 180(3):944-948.

18. F.Sanchez Mazzaferri, J.L.Fadil Iturralde. Fourniers gangrene. Our experience over 10 years. A review of the literature. Arch Esp Urol 1999, 52(7):721-727.
19. R.Zaba, A.Grzybowski, J.Prokop,et al. Fourniers gangrene: historical survey, current status, and case description. Med Sci Monit 2009, 15(2):CS34-CS39. 